\title{
Sonophotocatalysis of endocrine-disrupting chemicals
}

\author{
Toshinobu Tokumoto $^{\mathrm{a}, *}$, Katsutoshi Ishikawa ${ }^{a}$, Tsubasa Furusawa ${ }^{\mathrm{b}}$, Sanae Ii ${ }^{\text {a }}$, Kaori Hachisuka ${ }^{\mathrm{a}}$, \\ Mika Tokumoto ${ }^{a}$, Huai-Jen Tsai ${ }^{\mathrm{c}}$, Shigeo Uchida ${ }^{\mathrm{b}, 1}$, Akinori Maezawa $^{\mathrm{b}}$ \\ a Department of Biology, Faculty of Science, National University Corporation Shizuoka University, Shizuoka 422-8529, Japan \\ ${ }^{\mathrm{b}}$ Department of Materials Science and Chemical Engineering, Shizuoka University, 3-5-1, Johoku, Hamamatsu 432-8561, Japan \\ ${ }^{\mathrm{c}}$ Institute of Molecular and Cellular Biology, National Taiwan University, No. 1, Roosevelt Road, Sec. 4, Taipei 106, Taiwan
}

\section{A R T I C L E I N F O}

\section{Article history:}

Received 21 November 2007

Received in revised form 19 May 2008

Accepted 29 May 2008

\section{Keywords}

Endocrine-disrupting chemical

Decomposition of EDC

Photocatalysis

Sonolysis

Oocyte maturation

Zebrafish

\begin{abstract}
A B S T R A C T
Sonolysis and photolysis often exhibit synergistic effects in the degradation of organic molecules. An assay of fish oocyte maturation provides an appropriate experimental system to investigate the hormonal activities of chemical agents. Oocyte maturation in fish is triggered by maturation-inducing hormone $(\mathrm{MIH})$, which acts on receptors on the oocyte surface. A synthetic estrogen, diethylstilbestrol (DES), possesses inducing activity of fish oocyte maturation, and a widely used biocide, pentachlorophenol (PCP), exhibits a potent inhibitory effect on fish oocyte maturation.

In this study, the effects of the combined treatment by sonolysis with photolysis (sonophotocatalysis) to diminish the hormonal activity of DES and the maturation preventing activity of PCP was examined. By sonophotocatalysis, hormonal activity of DES was completely lost within $30 \mathrm{~min}$ and the inhibiting activity of PCP was lost within 120 min. These results demonstrated that sonophotocatalysis is effective for diminishing the endocrine-disrupting activity of chemical agents.
\end{abstract}

(c) 2008 Elsevier Ltd. All rights reserved.

\section{Introduction}

Recently, the treatment of wastewater containing organic carbon has been one of the most important subjects in environmental protection. A rapidly increasing number of chemicals, or their degradation products, are being recognized as possessing estrogenic activity, albeit usually weak. It has been found that effluent from sewage treatment works contains a chemical, or mixture of chemicals, that induces vitellogenin synthesis in male fish maintained in the effluent, thus indicating that the effluent is estrogenic (Sumpter and Jobling, 1995). Thus, the development of effective treatment technologies for eliminating chemical agents from the waste stream at its source is now the subject of considerable concern.

An assay of fish oocyte maturation provides an appropriate experimental system to investigate the hormonal actions of chemical agents. Several factors responsible for the regulation of oocyte maturation in fresh water fish have been identified. These include the isolation and characterization of a fish maturation-inducing hormone $(\mathrm{MIH}), 17 \alpha, 20 \beta$-dihydroxy-4-pregnen-3-one $(17,20 \beta-$

\footnotetext{
* Corresponding author. Address: Department of Biology, Faculty of Science, National University Corporation Shizuoka University, Oya 836, Suruga-ku, Shizuoka 422-8529, Japan. Tel.: +8154238 4778; fax: +81542384778.

E-mail address: sbttoku@ipc.shizuoka.ac.jp (T. Tokumoto).

1 Present address: Polytechnic College Hamamatsu, 693 Norieda-cho, Hamamatsu 432-8053, Japan.
}

DHP) (Nagahama et al., 1995), and the components of maturation-promoting factor (MPF) (cdc2, the catalytic subunit, and cyclin $\mathrm{B}$, the regulatory subunit) (Yamashita et al., 1995). Oocyte maturation in fish is triggered by $\mathrm{MIH}$, which acts on progestin receptors located on the oocyte membrane and induces the activation of MPF in the oocyte cytoplasm (Tokumoto et al., 2007). During the course of maturation, oocytes undergo drastic morphological changes associated with the progression of the meiotic cell cycle, among which breakdown of the oocyte nuclear envelope (germinal vesicle breakdown, GVBD) occurring at the prophase/metaphase transition is usually regarded as a hallmark of the progress of oocyte maturation.

Several endocrine-disrupting chemicals or EDCs, such as Kepon and $o, p$-DDD, have been reported to antagonize MIH-induced meiotic maturation of fish oocytes in vitro (Das and Thomas, 1999). EDCs such as methoxychlor and ethynyl estradiol also antagonize frog oocyte maturation. One EDC, diethylstilbestrol (DES), is a nonsteroidal substance, which was prescribed during the late 1940 s to early 1970s to pregnant women to prevent abortion, preeclampsia, and other complications of pregnancy. Male and female offspring exposed in utero to DES may develop multiple dysplastic and neoplastic lesions of the reproductive tract, along with other changes, during development (Bern, 1992). In a previous study, we found that treatment of oocytes with DES alone induced maturation in goldfish and zebrafish (Tokumoto et al., 2004). The maturationinducing activity of DES was also reported in a marine fish, goby (Baek et al., 2007). Furthermore, a potent inhibitory effect of 
pentachlorophenol (PCP) was demonstrated on oocyte maturation induced by MIH and DES (Tokumoto et al., 2005). PCP is a widely used biocide that has been employed as a wood preservative, herbicide, and defoliant. Its extensive use and persistence have resulted in significant environmental contamination and potential exposure of the general population (Crosby et al., 1981). Due to its highly persistent nature, PCP is still one of the dominant phenolic compounds in blood (Sandau et al., 2002). Recently, it was demonstrated that PCP was resistant for sulfonation, which is an important pathway in the biotransformation of a wide range of endogenous compounds and xenobiotics, in polar bears (Sacco and James, 2005). The carcinogenic effects of PCP have been evaluated in several animal bioassays (McConnell et al., 1991), and PCP levels in fish are used as a biomarker of contamination (Rogers et al., 1990). A relationship between PCP levels in women with reproductive and other endocrine problems was reported (Guvenius et al., 2003; Peper et al., 1999). Thus, PCP is an important organic chemical for environmental studies and various kinds of degradation technologies for PCP have been tried.

Photocatalysis, is a harmless wastewater purification method. An ultraviolet light source and titanium oxide photocatalyst are commonly used in the photocatalytic process. Ultraviolet light has a disinfectant effect and decomposes organic compounds to inorganic materials such as carbon dioxide and water. Some researchers have studied the decomposition of dye by photocatalysis (Alessandra et al., 2001; Sökem and Özkan, 2002). However, the decomposition efficiency is low, when the organic concentration in the wastewater is high.

The decomposition of organic compounds using ultrasound (called sonolysis) is harmless purification method. Several researchers have studied the ultrasonic decomposition of organic compounds such as alkyl ether (Suzuki et al., 1999), phenol (Weavers et al., 2005; Vassilakis et al., 2004), chlorofluorocarbon (Nagata et al., 1995), organohalogen compounds (Shemer and Narkis, 2004; Wu et al., 2001), and azo dye (Okitsu et al., 2005). One of the problems with sonolysis is its low decomposition efficiency. However, it has been reported by several researchers that the decomposition efficiency increased with the combination of photocatalysis and sonolysis (sonophotocatalysis) (Maezawa et al., 2007; Entezari et al., 2005; Chen and Smirniotis, 2002;Suzuki et al., 2000). Some of the reasons for the enhancement of decomposition efficiency are reported as follows:

(1) Catalyst particles are physically dispersed by ultrasonic treatment (Shirgaonkar and Pandit, 1998; Davydov et al., 2001; Ragaini et al., 2001).

(2) Enhancement of mass transfer between the bulk liquid and the surface of the catalyst and renewal of the fluid film near the surface of the catalyst (Shirgaonkar and Pandit, 1998; Stock et al., 2000).

(3) Formation of $\mathrm{OH}$ radicals from hydrogen peroxide, which is produced by the photocatalyst (Théron et al., 1999; Selli, 2002).

In the present study, the effectiveness of sonophotocatalysis in diminishing the hormonal activity of DES and antagonistic activity of PCP on fish oocyte maturation as a model of water pollutant chemicals was examined.

\section{Materials and methods}

\subsection{Materials}

The transgenic line TG ( $\beta$-actin:EGFP) was established by Hsiao and Tsai (2003). Although the cDNA integrated in this strain was constructed for expression of EGFP driven by the medaka $\beta$-actin promoter, the expression of EGFP is restricted to the oocytes in adult fish. Furthermore, the fluorescence is concentrated around the nucleus of immature oocytes (germinal vesicle), and diffuses throughout the mature oocyte after germinal vesicle breakdown (GVBD). TG zebrafish were bred and maintained at $28.5^{\circ} \mathrm{C}$ on a $14 \mathrm{~h}$ light/10 h dark cycle (Westerfield, 1995). 17,20ß-DHP and DES were purchased from Sigma Chemical Co. (St. Louis, MO, USA). Pentachlorophenol was obtained from Wako Pure Chemical Industries (Osaka, Japan).

\subsection{Sonophotocatalysis}

Details of the reactor for sonophotolysis were described previously (Maezawa et al., 2007). Major components of the reactor were following. A low-pressure mercury lamp (Sen Tokushu Kougen Co. Japan, UVL20PS) was used as a light source. The main emission wavelength is $185 \mathrm{~nm}$. The emission intensity of $254 \mathrm{~nm}$ is much lower than that of $185 \mathrm{~nm}$. Fixed $\mathrm{TiO}_{2}$ catalyst prepared from titanium isopropoxide by dip-coating method was used as a photocatalyst. As the ultrasonic transducer, the $250 \mathrm{kHz}$ commercial equipment from Kaijo Co. (Japan) was used. One liter of zebrafish Ringer's solution $\left(116 \mathrm{mM} \mathrm{NaCl}, 2.9 \mathrm{mM} \mathrm{KCl}, 1.8 \mathrm{mM} \mathrm{CaCl}_{2}\right.$, and 5 mM HEPES, pH 7.2) was poured into the reactor. Test agents were dissolved in zebrafish Ringer's solution at $10 \mu \mathrm{M}$ from a $10,000-$ fold stock in ethanol by stirring for $3 \mathrm{~min}$. Sonophotocatalysis was carried out for $120 \mathrm{~min}$ under conditions of stirring at $500 \mathrm{rpm}$ and aeration at $50 \mathrm{ml} / \mathrm{min}$. During the treatment about $20 \mathrm{ml}$ per time point of the solution was collected at $0,5,30,60$, and $120 \mathrm{~min}$. Samples were stored at $-20^{\circ} \mathrm{C}$ until use.

\subsection{Spectral analysis}

The changes in compounds during the sonophotocatalysis were evaluated by the absorption intensity between 220 and $340 \mathrm{~nm}$ using a UV-vis spectrophotometer (UV-2450, Hitachi Co., Japan).

\subsection{Oocyte preparation and in vitro culture}

Gravid female zebrafish, which possesses full-grown immature oocytes were selected from a group of mixture of 10-50 male and female that were kept in $20 \mathrm{~cm} \times 25 \mathrm{~cm}$ square and $25 \mathrm{~cm}$ high acryl case with continuous out-flow water. Zebrafish were sacrificed within two hours after lights were turned on. Ovaries were isolated from sacrificed females and placed in fresh zebrafish Ringer's solution (116 mM NaCl, $2.9 \mathrm{mM} \mathrm{KCl}, 1.8 \mathrm{mM} \mathrm{CaCl}_{2}$, and $5 \mathrm{mM}$ HEPES, $\mathrm{pH}$ 7.2) and washed with the same solution. Ovaries were dissected into ovarian fragments (each containing 2-10 oocytes) manually by using fine forceps. Fully-grown immature oocytes were exposed in vitro by incubating ovarian fragments in $4 \mathrm{ml}$ of zebrafish Ringer's solution containing each agent (from a 1000-fold stock in ethanol) at $25.0^{\circ} \mathrm{C}$ or room temperature with gentle agitation ( $40 \mathrm{rpm}$ ). GVBD was assessed by scoring the oocytes that became transparent (Pang and Ge, 2002) or scoring the GFPpositive GVs using a fluorescence microscope (SZX12, Olympus, Japan). \%GVBD was determined in more than twenty oocytes. The morphology of oocytes was recorded in photographs using a digital microscope (DP70, Olympus, Japan).

\subsection{Statistical analysis}

Summary data are presented as means \pm SD. The significance between multiple groups of data was evaluated using analysis of variance (ANOVA). Statistically significant differences between the percentage of GVBD were indicated as ${ }^{*} P<0.05$ or ${ }^{* *} P<0.01$. 


\section{Results and discussion}

Fig. 1 shows the morphology of oocytes after three hours treatment with ethanol (EtOH), 17,20 $\beta$-DHP, DES, and 17,20 $\beta$-DHP with PCP. Panels on the left show the images under normal lighting and panels on the right show fluorescence images. Previously, a transgenic zebrafish line with fluorescent oocytes was produced (Hsiao and Tsai, 2003). As shown in the figure, fluorescence in the transgenic oocytes is concentrated on the periphery of germinal vesicles. GVBD in this strain is clearly observed using the fluorescence microscope. Germinal vesicles were seen near the center of oocytes after EtOH and 17,20 $\beta$-DHP with PCP treatment, whereas the signal disappeared after the 17,20 $\beta$-DHP and DES treatments (Fig. 1B, D, F, and H). Also, 17,20 3 -DHP and DES treated oocytes became transparent (Fig. 1C, and E). DES induced GVBD in zebrafish oocytes in the same manner as the naturally occurring hormone 17,20 $\beta$-DHP. As shown in a previous report PCP completely prevented oocyte maturation. These compounds induce and inhibit oocyte maturation almost completely at concentrations of $10 \mu \mathrm{M}$.
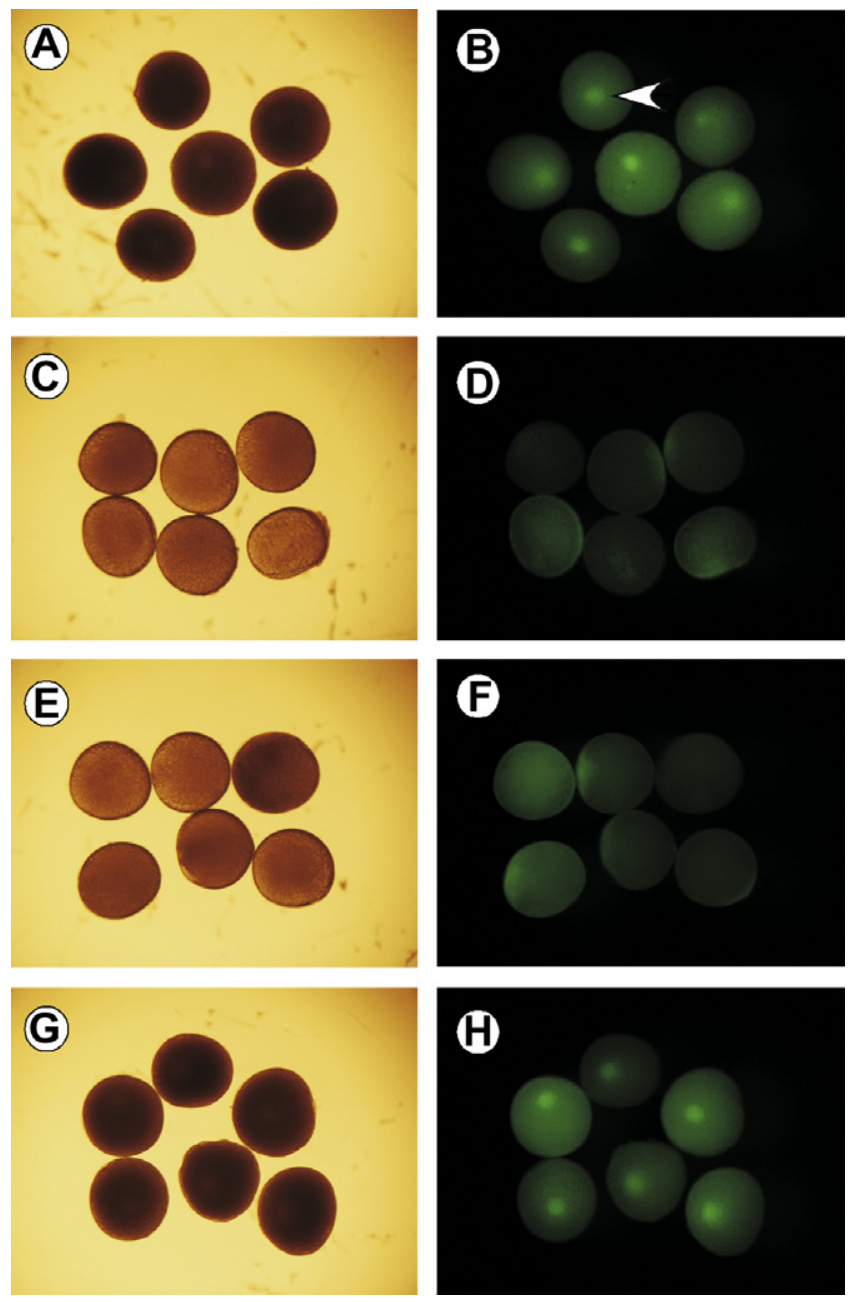

Fig. 1. GVBD observation in transgenic zebrafish. The morphology of oocytes after three hours of each treatment was recorded. Panels on the left indicate oocytes in zebrafish Ringer's solution. In panels on the right, oocytes were fixed in clearing solution for observation of germinal vesicles. Oocytes remained opaque after EtOH treatment, whereas they became transparent after $17 \alpha, 20 \beta$-DHP or DES treatments. Germinal vesicles were seen near the center of oocytes after EtOH treatment, whereas they disappeared after $17,20 \beta$-DHP or DES treatments. The arrowhead indicates a germinal vesicle. The scale bar represents $1 \mathrm{~mm}$.
To analyze the effect of sonophotocatalysis on hormonal activity of DES, DES-containing Ringer's solution was treated in a sonophotocatalysis chamber for $120 \mathrm{~min}$ and aliquots of the solution were collected at various times as samples for biological assaying. Oocytes were cultured in the samples of treated Ringer's solution. The sample collected before treatment (time 0) induced GVBD almost as completely as freshly prepared DES- and DHP-containing Ringer's solution. This result indicates that the hormonal activity of DES was stable in frozen Ringer's solution. As shown in Fig. 2A, the GVBD-inducing activity of DES was greatly reduced even by 5 min of treatment. The hormonal activity was almost lost after 30 min of treatment. When 17,20 3 -DHP was added into sample, that had been treated for $120 \mathrm{~min}$, the full magnitude of GVBD was induced. This result demonstrated that the decrease of GVBDinducing activity of treated samples was not derived from the production of toxic substances by sonophotocatalysis, but was due to the breakdown of DES. This is supported by spectral analysis (Fig. 2B). Changes in the spectral pattern of samples, which suggested the degradation of DES, occurred after $5 \mathrm{~min}$. These results indicate that DES was degraded by sonophotocatalysis rapidly and lost its hormonal activity. As described previously for compounds that lack ethyl groups and when hydroxyls were blocked by substitution, the hormonal activity of DES was greatly reduced. This result suggested that the structural changes following by sonophotocatalysis caused a reduction in the activity of DES. As expected, the activity of DES reduced within a short period.

In contrast to DES, a relatively long time was needed to degrade PCP for diminish its activity. As shown in Fig. 3, the inhibitory effect of PCP was begun to be lost after 60 min of treatment and lost completely after $120 \mathrm{~min}$. This was supported by the spectral analysis (Fig. 3B). Changes in the spectral pattern of samples suggested that the degradation of PCP occurred at $60 \mathrm{~min}$. The transient peak at $280-300 \mathrm{~nm}$ could be derived from an intermediate of PCP degradation process as this peak decreased to $50 \%$ at $60 \mathrm{~min}$ and disappeared within $120 \mathrm{~min}$. A correlation between the peak area and the inhibiting activity of the treated PCP solution was observed. This suggested that intermediates of PCP in the degradation process still possess inhibiting activity and 120 min was necessary to degrade PCP to inactive compounds. When oocytes were incubated in sample that was treated for $120 \mathrm{~min}$, GVBD was not induced. This result demonstrated that the increase in GVBD-inducing activity of treated samples was not derived from the production of hormonally active substances by sonophotocatalysis of PCP. These results indicate that PCP was degraded by sonophotocatalysis and lost its inhibitory activity.

There have been various studies on the elimination of hormonally active chemicals from wastewater. Coleman et al. (2004) reported the degradation of estrogens by UVA photolysis and photocatalysis over an immobilized titanium dioxide $\left(\mathrm{TiO}_{2}\right)$ catalyst. They studied the loss of estrogenic activity of steroid estrogens constituting the main estrogenic component, $17 \beta$-estradiol (E2) and estrone (E1), and the synthetic steroid estrogen $17 \alpha$-ethinylestradiol (EE2), in domestic sewage treatment work (STW) effluent. Estrogenic compounds lost their hormonal activity in a short period by photocatalysis over an immobilized $\mathrm{TiO}_{2}$ catalyst. The results demonstrated that photocatalysis is effective at degrading estrogenic compounds.

PCP has been widely used as a wood preservative, herbicide, and insecticide all over the world. This has resulted in ubiquitous environmental pollution and the hazard risk of human exposure is now a matter of concern (Vartiainen et al., 1995). Biodegradation of PCP in water is slow and incomplete. There have been report on a variety of approaches to degrade and remove PCPs from environment. For example biodegradation by bacteria, or fungus, or enzymes isolated from these organisms, has been reported ( $\mathrm{Wu}$ et al., 1993; Ye and Li, 2007). Although PCP is capable of biodegra- 

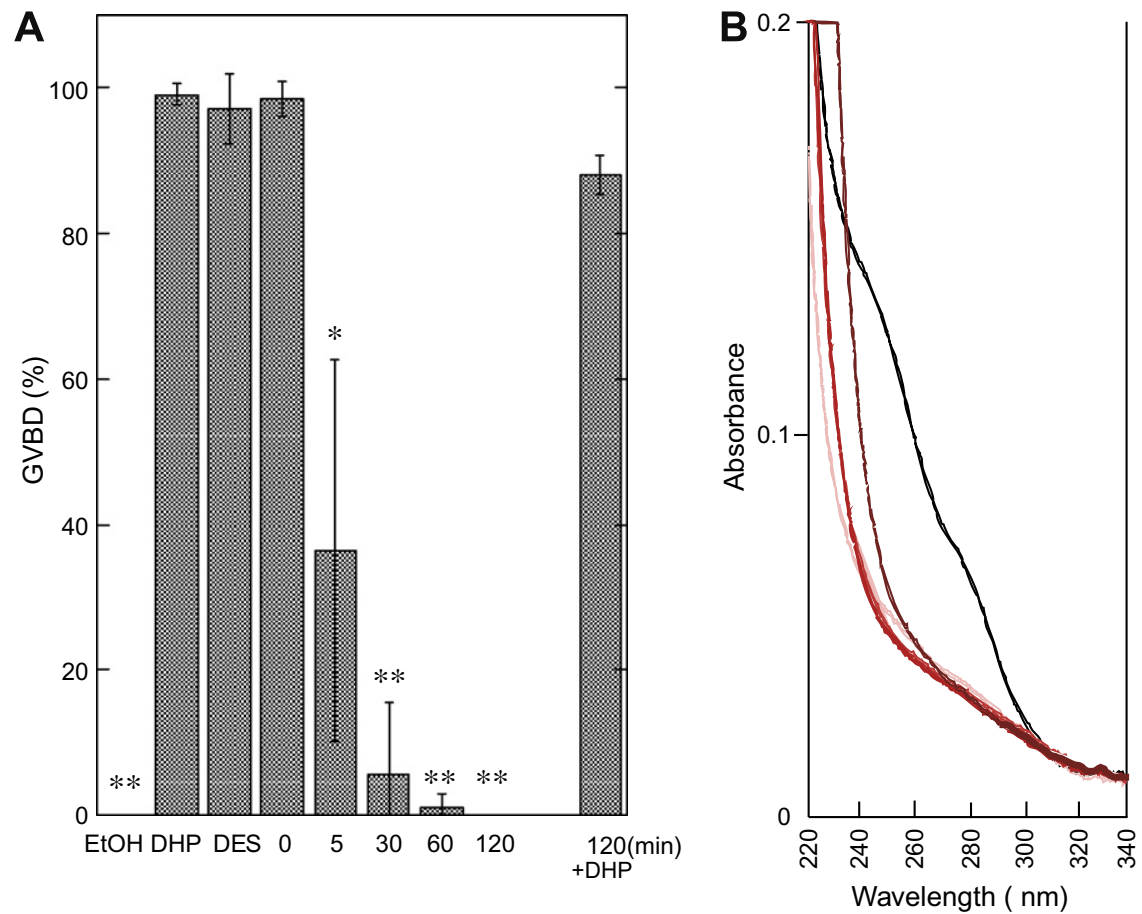

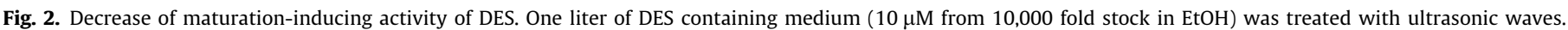

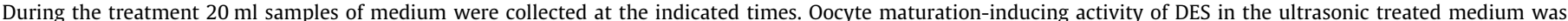

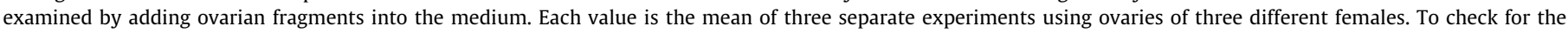

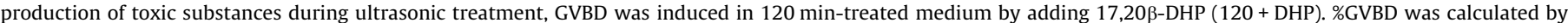

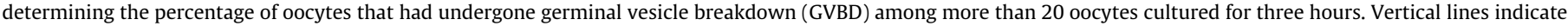

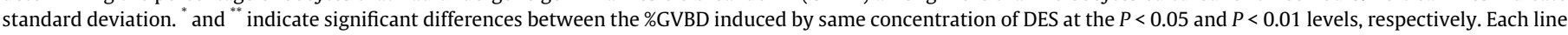
in panel B represents the following times: —, $0 \mathrm{~min} ; \_, 5 \mathrm{~min} ;-, 30 \mathrm{~min} ;-, 60 \mathrm{~min} ; \_, 120$ min. (Please see electronic version for colour reproduction).
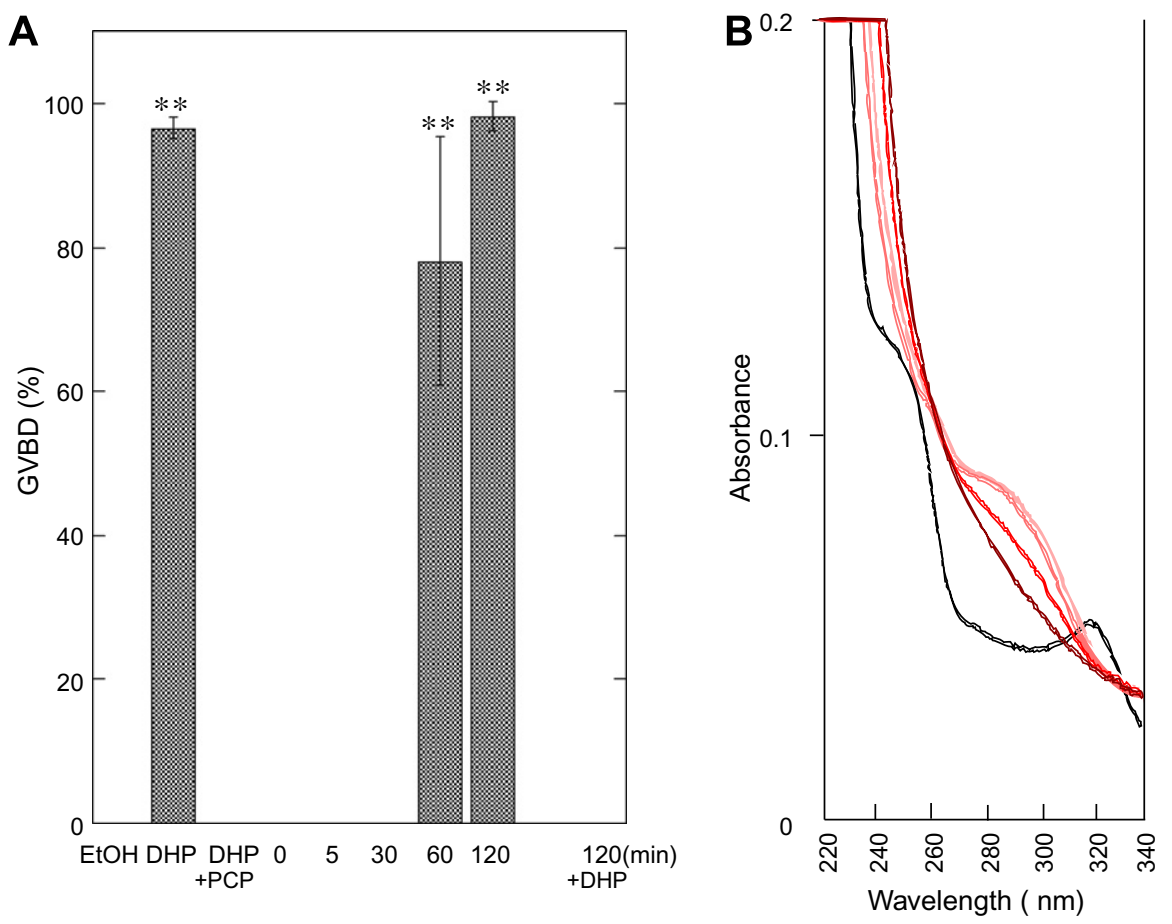

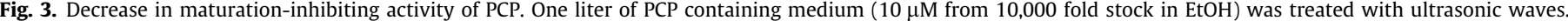

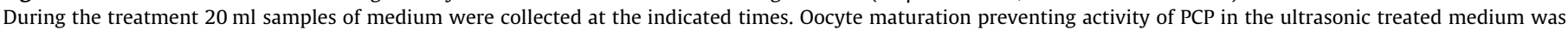

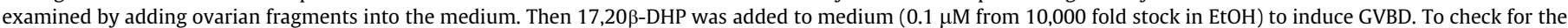

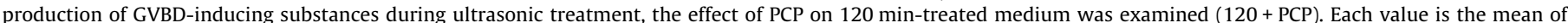

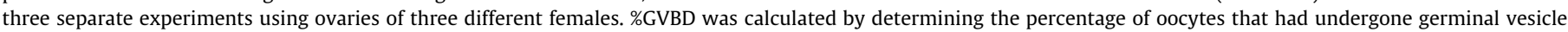

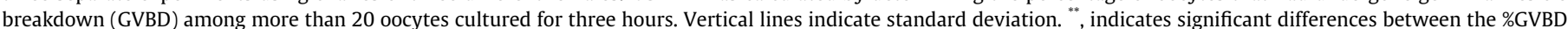

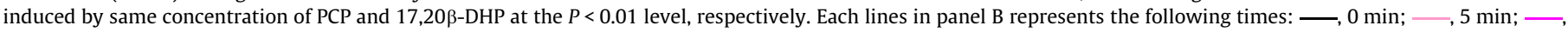
$30 \mathrm{~min} ; \_, 60 \mathrm{~min} ; \_, 120 \mathrm{~min}$. (Please see electronic version for colour reproduction). 
dation, the kinetics to complete its mineralization are slow. More recently, advanced oxidation processes were attempted for the degradation of PCPs such as ozonation, photolysis, or photocatalysis (Fukushima and Tatsumi, 2007; Hanna et al., 2004; Hong and Zeng, 2002; Quan et al., 2007). Hong and Zeng determined chemical structure of intermediates during degradation of PCP by ozonation in different $\mathrm{pH}$ conditions (2002). They demonstrated that 2,3,5,6-tetrachloro-p-hydroquinone is an abundant intermediate at $\mathrm{pH}$ 7.0. Shoulder peak at $280-300 \mathrm{~nm}$ appeared during sonophotolysis of PCP in this study corresponded to the peak of the intermediate. It seems likely that the intermediate was produced during sonophotolysis and possessed inhibitory activity against induction of oocyte maturation. On the other hand, effective treatment technologies for eliminating chemical agents from wastewater have been developed. A recently developed technology, sonophotocatalysis, is an effective and economical method to degrade organic compounds in solution (Selli, 2002; Maezawa et al., 2007). In the present study, the effectiveness of sonophotocatalysis for eliminating hazardous materials in the water was evaluated by a well-established biological assay.

\section{Conclusions}

It was demonstrated that DES lost its hormonal activity in a very short period and PCP was degraded and lost its inhibiting activity on fish oocyte maturation within $120 \mathrm{~min}$. In sonophotocatalysis, undesirable production of compounds that have inhibiting or inducing activity on oocyte maturation was undetectable during DES and PCP degradation. As shown in this study, sonophotocatalysis is an effective technology for eliminating chemical agents, especially for endocrine-disrupting chemicals.

\section{Acknowledgements}

This work was supported by Grants-in-Aid for Scientific Research on Priority Areas from the Ministry of Education, Culture, Sports, Science, and Technology of Japan (16360393 to S.U. and 17570175 to T.T.).

\section{References}

Alessandra, B.P., Claudio, B., Maria, C.B., 2001. Photocatalytic degradation of acid blue 80 in aqueous $\mathrm{TiO}_{2}$ suspentions. Environmental Science and Technology 35, 971-976.

Baek, H.J., Hwang, I.J., Kim, K.S., Lee, Y.D., Kim, H.B., Yoo, M.S., 2007. Effects of BPA and DES on longchin goby (Chasmichthys dolichognathus) in vitro during the oocyte maturation. Marine Environmental Research 64, 79-86.

Bern, H.A., 1992. Chemically Induced Alterations in Sexual and Functional Development: The Wildlife/Human Connection. Princeton Sci. Pub., Prinston, NJ.

Chen, Y.-C., Smirniotis, P., 2002. Enhancement of photocatalytic degradation of phenol and chlorophenols by ultrasound. Industrial and Engineering Chemistry Resarch 41, 5958-5965.

Coleman, H.M., Routledge, E.J., Sumpter, J.P., Eggins, B.R., Byrne, J.A., 2004. Rapid loss of estrogenicity of steroid estrogens by UVA photolysis and photocatalysis over an immobilised titanium dioxide catalyst. Water Research 38, 3233-3240.

Crosby, D.J., Beynon, K.L., Greve, P.A., Korte, F., Stili, G.G., Vouk, J.W., 1981. Environmental chemistry of pentachlorophenol. Pure and Applied Chemistry 53, 1051-1081.

Das, S., Thomas, P., 1999. Pesticides interfere with the nongenomic action of a progestogen on meiotic maturation by binding to its plasma membrane receptor on fish oocytes. Endocrinology 140, 1953-1956.

Davydov, L., Reddy, E.P., France, P., Smirniotis, P.G., 2001. Sonophotocatalytic destruction of organic contaiminants in aqueous systems on $\mathrm{TiO}_{2}$ powders. Applied Catalysis B: Environmental 32, 95-105.

Entezari, M.H., Heshmari, A., Sarafraz-yazdi, A., 2005. A combination of ultrasound and inorganic catalyst: removal of 2-chlorophenol from aqueous solution. Ultrasonics Sonochemistry 12, 137-141.

Fukushima, M., Tatsumi, K., 2007. Degradation of pentachlorophenol in contaminated soil suspensions by potassium monopersulfate catalyzed oxidation by a supramolecular complex between tetra(psulfophenyl)porphineiron(III) and hydroxypropyl-beta-cyclodextrin. Journal of Hazardous Materials 144, 222-228.
Guvenius, D.M., Aronsson, A., Ekman-Ordeberg, G., Bergman, A., Noren, K., 2003. Human prenatal and postnatal exposure to polybrominated diphenyl ethers, polychlorinated biphenyls, polychlorobiphenylols, and pentachlorophenol. Environmental Health Perspectives 111, 1235-1241.

Hanna, K., de Brauer, C., Germain, P., Chovelon, J.M., Ferronato, C., 2004. Degradation of pentachlorophenol in cyclodextrin extraction effluent using a photocatalytic process. Science of the Total Environment 332, 51-60.

Hong, P.K., Zeng, Y., 2002. Degradation of pentachlorophenol by ozonation and biodegradability of intermediates. Water Research 36, 4243-4254.

Hsiao, C.D., Tsai, H.J., 2003. Transgenic zebrafish with fluorescent germ cell: a useful tool to visualize germ cell proliferation and juvenile hermaphroditism in vivo. Developmental Biology 262, 313-323.

Maezawa, A., Nakadoi, H., Suzuki, K., Furusawa, T., Suzuki, Y., Uchida, S., 2007. Treatment of dye wastewater by using photo-catalytic oxidation with sonication. Ultrasonics Sonochemistry 14, 615-620.

McConnell, E.E., Huff, J.E., Hejtmancik, M., Peters, A.C., Persing, R., 1991. Toxicology and carcinogenesis studies of two grades of pentachlorophenol in B6C3F1 mice. Fundamental and Appllied Toxicology 17, 519-532.

Nagahama, Y., Yoshikuni, M., Yamashita, M., Tokumoto, T., Katsu, Y., 1995 Regulation of oocyte growth and maturation in fish. Cellular and Molecular Procedures In Developmental Biology 30, 103-145.

Nagata, Y., Hiraki, K., Okitsu, K., Dohmaru, T., Maeda, Y., 1995. Decomposition of chlorofluorocarbon CFC-113 in water by ultrasonic irradiation. Chemistry Letters, 203-204.

Okitsu, K., Iwasaki, K., Yobiko, Y., Bandow, H., Nishimura, R., Maeda, Y., 2005. Sonochemical degradation of azo dyes in aqueous solution: a new heterogeneous kinetics model taking into account the local concentration of $\mathrm{OH}$ radicals and azo dyes. Ultrasonics Sonochemistry 12, 255-262.

Pang, Y., Ge, W., 2002. Gonadotropin and activin enhance maturational competence of oocytes in the zebrafish (Danio rerio). Biology of Reproduction 66, 259-265.

Peper, M., Ertl, M., Gerhard, I., 1999. Long-term exposure to wood-preserving chemicals containing pentachlorophenol and lindane is related to neurobehavioral performance in women. American Journal of Industrial Medicine 35, 632-641.

Quan, X., Ruan, X., Zhao, H., Chen, S., Zhao, Y., 2007. Photoelectrocatalytic degradation of pentachlorophenol in aqueous solution using a $\mathrm{TiO}_{2}$ nanotube film electrode. Environmental Pollution 147, 409-414.

Ragaini, V., Selli, E., Bianchi, C.L., Pirola, C., 2001. Sono-photocatalytic degradation of 2-chlorophenol in water: kinetic and energetic comparison with other techniques. Ultrasonics Sonochemistry 8, 251-258.

Rogers, I.H., Birtwell, I.K., Kruzynski, G.M., 1990. The Pacific eulachon (Thaleichthys pacificus) as a pollution indicator organism in the Fraser River estuary, Vancouver, British Columbia. Science of the Total Environment, 713-727.

Sacco, J.C., James, M.O., 2005. Sulfonation of environmental chemicals and their metabolites in the polar bear (Ursus maritimus). Drug Metabolism and Disposition 33, 1341-1348.

Sandau, C.D., Ayotte, P., Dewailly, E., Duffe, J., Norstrom, R.J., 2002. Pentachlorophenol and hydroxylated polychlorinated biphenyl metabolites in umbilical cord plasma of neonates from coastal populations in Quebec. Environmental Health Perspectives 110, 411-417.

Selli, E., 2002. Synergistic effects of sonolysis combined with photocatalysis in the degradation of an azo dye. Physical Chemistry Chemical Physics 4, 6123-6128.

Shemer, H., Narkis, N., 2004. Mechanism and inorganic byproducts of trihalomethane compounds sonodegradation. Environmental Science and Technology 38, 4856-4859.

Shirgaonkar, I.Z., Pandit, A.B., 1998. Sonophotochemical destruction of aqueous solution of 2,4,6-trichlorophenol. Ultrasonics Sonochemistry 5, 53-61.

Sökem, M., Özkan, A., 2002. Decolouring textile wastewater with modified titania: the effect of inorganic anions on the photocatalysis. Journal of Photochemistry Photobiology A: Chemistry 147, 77-81.

Stock, N.L., Peller, J., Vinodgopal, K., Kamat, P.V., 2000. Combinative sonolysis and photocatalysis for textile dye degradation. Environmental and Science Technology 34, 1747-1750.

Sumpter, J.P., Jobling, S., 1995. Vitellogenesis as a biomarker for estrogenic contamination of the aquatic environment. Environmental Health Perspectives 103 (Suppl. 7), 173-178.

Suzuki, Y., Maezawa, A., Uchida, S., 1999. Effect of frequency and aeration rate on ultrasonic oxidation of surfactant. Chemical Engineering and Technology 22, 507-510.

Suzuki, Y., Maezawa, A., Uchida, S., 2000. Utilization of ultrasonic energy in a photocatalytic oxidation process for treating waste water containing surfactant. Japanese Journal of Applied Physics 39, 2958-2961.

Théron, P., Pichat, P., Guillard, C., Pétrier, C., Chopin, T., 1999. Degradation of phenyltrifluoromethylketone in water by separate or simultaneous use of $\mathrm{TiO}_{2}$ photocatalysis and 30 or $515 \mathrm{kHz}$ ultrasound. Physical Chemistry Chemical Physics 1, 4663-4668.

Tokumoto, T., Tokumoto, M., Horiguchi, R., Ishikawa, K., Nagahama, Y., 2004. Diethylstilbestrol induces fish oocyte maturation. Proceedings of the National Academy of the Sciences of the United States of America 101, 3686-3690.

Tokumoto, T., Tokumoto, M., Nagahama, Y., 2005. Induction and inhibition of oocyte maturation by EDCs in zebrafish. Reproductive Biology and Endocrinology 3, 69.

Tokumoto, T., Tokumoto, M., Thomas, P., 2007. Interactions of diethylstilbestrol (DES) and DES analogs with membrane progestin receptor-alpha and the correlation with their nongenomic progestin activities. Endocrinology 148 3459-3467. 
Vartiainen, T., Lampi, P., Tuomisto, J.T., Tuomisto, J., 1995. Polychlorodibenzo-pdioxin and polychlorodibenzofuran concentrations in human fat samples in a village after pollution of drinking water with chlorophenols. Chemosphere 30 , 1429-1438.

Vassilakis, C., Pantidou, A., Psillakis, E., Kalogerakis, N., Mantzavinos, D., 2004 Sonolysis of natural phenolic compounds in aqueous solutions: degradation pathways and biodegradability. Water Research 38, 3110-3118.

Weavers, L.K., Pee, G.Y., Frim, J.A., Yang, L., Rathman, J.F., 2005. Ultrasonic destruction of surfactants, application to industrial waste water. Water Environment Research 77, 259-265.

Westerfield, M., 1995. The Zebrafish Book: A Guide for the Laboratory Use of Zebrafish (Danio rerio). University of Oregon Press, Eugene, OR.
Wu, W.M., Bhatnagar, L., Zeikus, J.G., 1993. Performance of anaerobic granules for degradation of pentachlorophenol. Applied and Environmental Microbiology 59, 389-397.

Wu, C., Lin, X., Wang, L., Fan, J., 2001. Ultrasonic destruction of Chloroform and carbon tetrachloride in aqueous solution. Journal of Environmental Science and Health Part A A 36, 947-955.

Yamashita, M., Kajiura, H., Tanaka, T., Onoe, S., Nagahama, Y., 1995. Molecular mechanisms of the activation of maturation-promoting factor during goldfish oocyte maturation. Developmental Biology 168, 62-75.

Ye, F.X., Li, Y., 2007. Biosorption and biodegradation of pentachlorophenol (PCP) in an upflow anaerobic sludge blanket (UASB) reactor. Biodegradation 18, 617624. 